\title{
A prototype of a social and economic based resource allocation system in grid computing
}

\author{
Gabriele Pierantoni, Eamonn Kenny, Brian Coghlan \\ Trinity College Dublin, Computer Architecture Group \\ Rm-005, CALab1, INS building, TCD, Dublin 2, Ireland \\ pierantg@cs.tcd.ie, ekenny@cs.tcd.ie, coghlan@cs.tcd.ie
}

\begin{abstract}
Nowadays the grid community is facing complex challenges. Among them three are particularly interesting: the existence of many different grid middlewares; the increasing complexity of the submitted jobs; and the many different ways in which actors and resources can be arranged. In this paper we propose a combined solution to these problems which is based on the union of two concepts: a metagrid concept that tackles the issues posed by the variety of different middlewares and the complexity of jobs, and a concept of Social Grid Agents that tackles the issue of resource sharing and allocation.
\end{abstract}

\section{Introduction}

Grid computing was born as an elegant way to bring together resources, their owners and their users in the digital world. By doing so, it allowed users to harness more resources than they owned and allowed resources to be more fully used than would otherwise have been possible.

Many different grid middlewares were developed, each with strengths and weaknesses. The users continue to request the execution of tasks of growing complexity and the social relationships among resource owners and consumers continue to become more and more intricate.

The grid community tries to cope with these problems by offering sophisticated grid middlewares. Particularly the growing complexity of the tasks submitted is tackled by the workflow engine field of research, the ever increasing number of different grid middlewares is coped with by the grid interoperability community and the increasing complexity in the resources allocation schemes is dealt with by research in the resource brokerage community. The various solutions[6],[11] envisaged by the scientific community to tackle the complexities of resource brokerage and allocation in grids encompass genetic algorithms, simulated anneal- ing and hybrid solutions[3]. In addition to these, there are economy-based approaches that range from pure competitive liberalism to pure cooperation and from uncontrolled markets[5][21] to centralized controlled economies[12][9]. Nonetheless, all of these enable in effect low-level (the majority) or mid-level (the minority) resource brokerage. In this paper we describe the architecture of the prototype of the solution we proposed for these three combined problems. Our solution, which we term, Social Grid Agents[2] has been described from an abstract and theoretical perspective, from a resource allocation perspective[16][17] and with regard to the issue of interoperability[20][19] and, finally, with regard to the growing number of different workflow engines[18]. The prototype allows us to elucidate the architecture in more detail.

\section{Paradigm used in the design of the Proto- type}

The paradigm that led us to the design of this prototype tries to cope with the problems listed in section 1 (multiple grids, complex jobs, multiple actors and different allocation scenarios). It is based on the assumption that these problems are similar in nature, if not in degree, to those that mankind has faced since its dawn: the management of limited resources to fulfill the increasingly complex needs of a variety of actors with different goals and priorities. Mankind strives to solve this problem with economic and social exchange and structures called societies where the members of the society can engage in a variety of allocation and exchange methods under a common set of rules. Moreover, the design of the prototype tries to cope with a set of other constraints, such as: the availability of many different grid middlewares (including workflow-centered) that must be offered to a user, The bewildering variety of different relationships among resource owners, institutions and users and the need for a flexible and scalable solution able to adapt to future middlewares with unknown characteris- 
tics.

\subsection{A socio-economic perspective of grid Computing}

The design we offer tries to cope with these complexities and constraints. It is based on three flows of information:

- The production chain : The starting point of the design was to model a grid as a microeconomic[15] production chain that spawns from basic goods, or factors, to intermediate commodities and, in the end, to the final job, or produced commodity. Existing grid resources (job submission, information systems and file management services) are, in reality, produced commodities but we treat them as factors as their internal production chain is not modeled directly in economic terms.

- The ownership flow: At each of the production steps an actor (either a human or a program) assembles factors and, possibly, intermediate commodities to create a produced commodity. If at any given time an actor has not enough factors to produce the commodities he wants he may engage in social or economic transactions to obtain the commodities he needs. These types of relations can be: co-operative relations consisting of access to resources without the need for a balancing transaction representing a payment such as donations, lending and borrowing, Keynesian investments ${ }^{1}$ and common goods ${ }^{2}$, Non-monetary economic relations such as bartering, and Monetary economic relations that involve payments such as Posted Price Model, Auctions and Calls for Tender.

- The policies flow: The production process and the social interactions are also bound to policies that constrain the way the agents engage in production or in social and economic exchange. Policies also define what kind of economic or co-operative agreements bind two or more agents.

\subsection{General Architecture}

The architecture is based on two layers as described in Figure 1; one where Production Grid Agents compose various grid services as in a microeconomic supply chain and another layer where the Social Grid Agents that own and control the agents in the lower layer engage in social and economic exchange.

\footnotetext{
${ }^{1}$ We term Keynesian Investment, in honor of John Maynard Keynes, a relationship where an Institution invests in grid resources binding their use, partially or fully, to a certain user or use

${ }^{2}$ We detail these two relationships in Section 3.
}

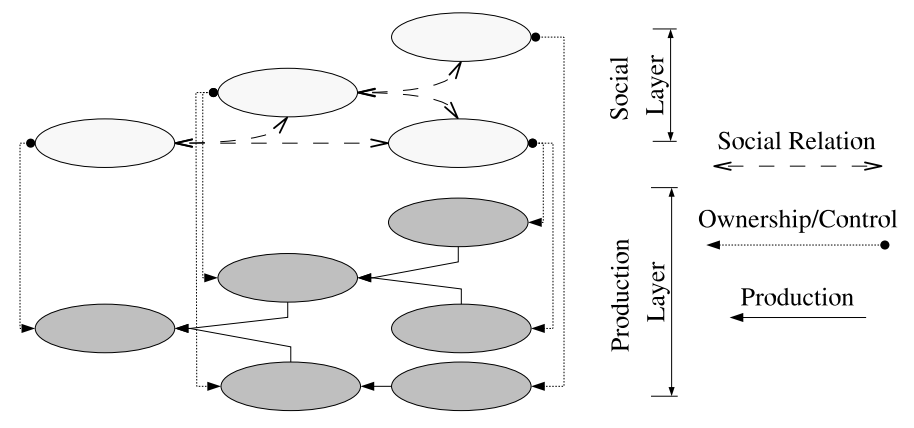

Figure 1. A socio-economic perspective of a grid.

This design allows for a large variety of connection topologies among the Social and Production Grid Agents. Each Social Grid Agent can own, control or temporarily use none, one or more Production Grid Agents. A Social Grid Agent can also exchange the control of, or the services produced by, the Production Grid Agent it controls. Therefore, the design of the agents had primarily to focus on how to handle the three flows of information (production, ownership and policies) in a consistent, flexible and scalable manner. This they do in a metagrid environment (see section 4) in order that a Production Agent can be anything from a single resource to an entire grid like EGEE[8].

\section{The Agent Design}

In order to achieve the design requirements and constraints listed in section 2, the agents must be able to assemble different resources and accept or reject requests from other agents depending on the relationship that binds them; moreover both the Production and Social Grid Agents should share a common design in order to reduce implementation and maintainance complexity. The architecture of the agents is composed from two or three main entities. Production Grid Agents that encompass existing grid resources (or Border Agents, see section 4) have a Grid Body consisting of existing grid services surrounded by a GT4 service ${ }^{3}$ that enable it to be used by the other metagrid components. The link between the GT4 service and the existing grid service usually consists of API invocations but can also be performed with system calls if an API is lacking. All other agents such as Native Metagrid Agents (again see section 4) or Social Grid Agents contain a Skeleton that consists of the services offered by the GT4 services. Finally, a Java Brain implements the production or social behavior with which the agent controls the existing grid service. The most complex of these components, the Java Brain, is described in

\footnotetext{
${ }^{3}$ GT4 is the Globus Toolkit version, see http://www.globus.org.
} 
detail in Figure 2.

All Java Brains expose a minimal interface composed of the method process(message) that returns another message. This important design decision was made in order to reduce to a minimum the complexity of the agent's skeleton. When a message is received by a Java Brain, its Processor Manager checks whether there is any Processor (PR in Figure 2) able to manage it. A Processor is a Java class that contains the "knowledge" of what to do with one or more messages. The processor will behave according to its current policies.

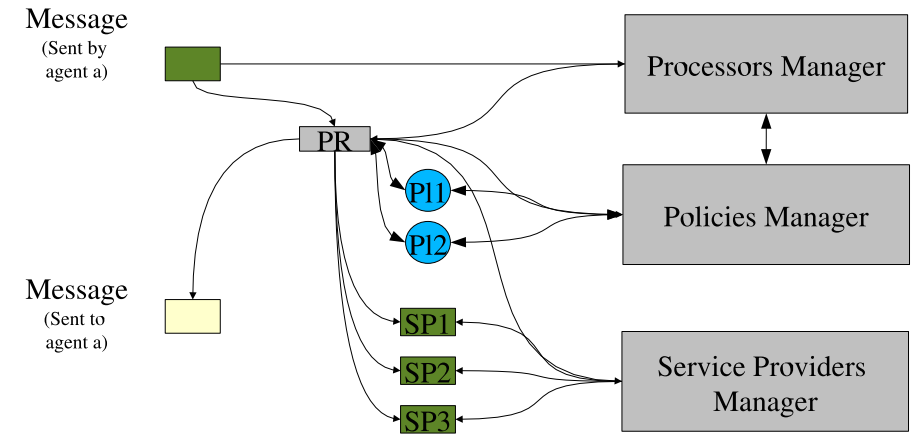

Figure 2. Agent Architecture.

The Processor Manager is capable of match-making a Processor to a message, its sender and a policy through hash maps. These maps are used to describe set relations between message types, values of the fields of the message, policies and Processors capable of handling them. If a Processor capable of handling that message with that policy is found, it is instantiated and it is passed the message. The Processor then analyzes the message and compiles a list of services it needs, which it then passes to the Service Providers Manager that instantiates the necessary Service Providers (SP in Figure 2) using a very similar mechanism to the Processor Manager. The Processor also queries the Policy Manager to obtain the policies that must be enforced during the execution.

The detailed working of this mapping technique is described in Figure 3. Firstly a message $m$ of type $t$ is received by the agent from a sender $s$. This message is part of a hierarchy of Java classes that implement all the messages accepted by the agents. The message also has some fields $v_{i}$ that are useful for the selection of the processor.

The first step in the mapping process is to link the sender to a relationship $s \rightarrow r$. Then the message type, the sender and the relationship map to a policy: $(t, s, r) \rightarrow p$. Finally the message type, the field values and the policy map to a processor: $\left(t,\left\{v_{i}\right\}, p\right) \rightarrow$ processor.

Agents must take different actions depending on the sender identity and the message; for example an agent may accept service execution requests for free from an agent it has a cooperative relationship with, it may ask for a stan-

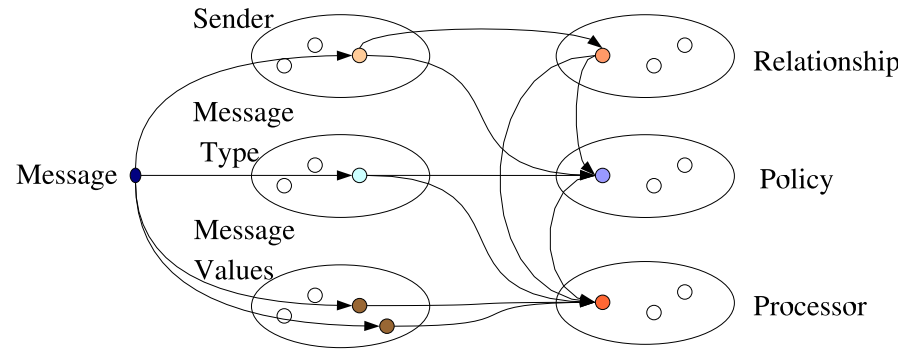

Figure 3. Mapping a message to a processor.

dard price for a different set of agents and have a particular set of prices for a third set of agents with whom it has a particular economic relationship. In order to achieve this flexible behavior every Policy Manager also deals with access rights, offering an AccessMap service that tells whether and how a certain message must be replied to by the agent. The AccessMap service behavior is described in Figure 4; it is also a good example to explain the behavior of the Policy Manager and all the managers in general. When a message $M s g_{i}$ is received from the Agent with identity $I d_{j}$ the message type and the identity are combined into a new object, the AccessKey $A k\left(M s g_{i}, I d_{j}\right)$. This AccessKey is then used as a key to a HashMap that contains the policies (in this case access modalities) which the agent must adhere to. Policies are arranged in type hierarchies stemming from an abstract class. Such policies mainly cover relationships between Social and Production Grid Agents (control and access modalities) or between Social Grid Agents (social and economic modalities).

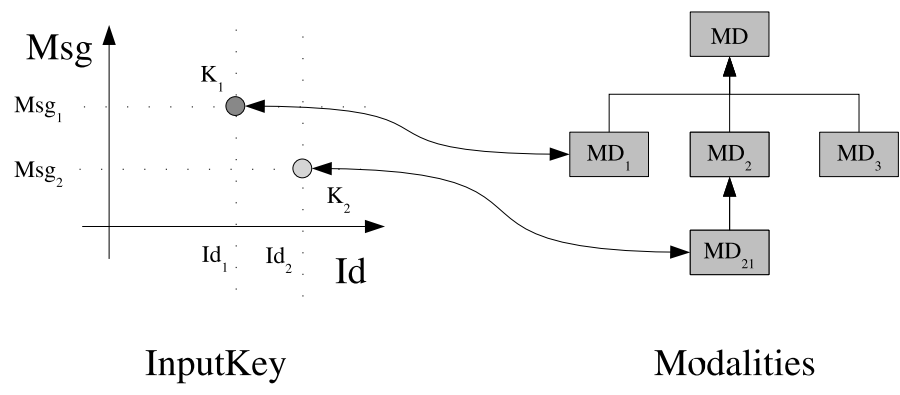

Figure 4. Access Map.

\subsection{Production control topologies}

As indicated above, policies and modalities are design features that enable agents to behave differently depending on the nature of their relationship with other agents. These are modeled as hierarchies of Java classes that expose a common interface. The access modalities hierarchy, 
for example, exposes a common method isGranted(sender, request) that returns True if the sender can submit a request to the agent. Some of the access modalities are:

- The Absolute Modality: Either True or False, it is mostly used to represent ownership relationships of Production Grid Agents.

- The Token Modality: Allows an Agent to submit a certain number of requests to a Production Grid Agent. It is mostly used in simple Service Rental scenarios where a Social Grid Agent buys (or receives) a given amount of service execution rights on Production Grid Agents it does not control or own.

- The Deadline Modality: Allows an Agent to control another agent for a given amount of time. It is mostly used in more complex Service Rental scenarios where a Social Grid Agents rents (or gets for free) control of other Production Grid Agents to engage in the execution of a complex service.

- The TimeSlot Modality: Allows an Agent to use the services of another agent until the allowed amount of time has been used. It is mostly used to reserve processing and storage services.

- The Combined Modalities: Some of the above mentioned modalities can be combined together. Token and Deadline modality can be combined to obtain a modality where tokens have expiry dates while TimeSlot and Deadline modalities can be combined to obtain a "perishable" reservation on computational and storage services.

Agents can have different roles with respect to a Production Grid Agent. These roles are: the owners that have complete control over a Production Grid Agent; they can sell, rent or donate the Production Grid Agent to other agents; they can set access rights for Controllers and Users and they can set policies for the Production Grid Agent, the controllers that have partial control and can set access rights for Users and set production policies, and the users that can access the services granted by a controller or an owner.

It is clear that some form of control has to be provided over the modalities with which each of the actors sets the rights of the others. If no such control is provided there may be cases in which agents gain control of other agents in an unforeseen way. As an example, let us suppose that an owner $\mathrm{O}$ grants control to an agent $\mathrm{C}$ to one of his production agents $\mathrm{P}$, with a deadline modality that expires at a date T. As the controller can set any kind of access rights for the user it is possible for the controller to set absolute rights to a user $\mathrm{U}$. These rights, being absolute, will outlive the range of time in which $\mathrm{C}$ is allowed to control $\mathrm{P}$. To avoid such problems the setting of the access rights are as follows.
- For owners: Owners can set any kind of access rights and they are enforced directly.

- For controllers: the access rights that controllers enforce depend on the access rights they have, specifically:

- Absolute modality: enforced directly.

- Deadline modality: If the controller has Deadline modality access rights, its commands change in the following way.

$*$ Absolute modality $\rightarrow$ Deadline modality.

$*$ Deadline modality $\rightarrow$ Deadline modality with an expiry date that is the minimum between the controller's and the target's settings.

$*$ TimeSlot modality $\rightarrow$ TimeSlot and Deadline modality.

* Token modality $\rightarrow$ Deadline and Token modality.

- Token modality: not used. Token modality is granted to users, not controllers.

- For users: Users cannot set any access right.

To allow flexibility at the startup of the container hosting the agents an "imprinting" mechanism has been designed. At startup an agent has a secret code. Any agent that knows that secret code is allowed to set itself or any other agent as Owner. This mechanism also allows it to sell or buy a Production Grid Agent. In this case the owner selling the Production Grid Agent to a client sets a new startup code, then it passes it to the client. This architecture allows many different production topologies. Three of these are now described.

\subsubsection{Simple Producer}

The simplest of these topologies is the Simple Producer shown in Figure 5 where a client contacts a Social Grid Agent and arranges the purchase or free acquisition of a grid resource (step 1). When the social or economic transaction is accepted by the two parties the Social Grid Agent gives instructions to the Production Grid Agent it controls to execute the required Service (step 2). Then the service is performed (step 3) and the results are sent back to the client (step 4 and 5). Obviously even such a simple example can be implemented in many different ways. From a social and economic perspective there may or may not be a payment required for the service depending on the relationship between the agents. There can be different payment policies such as beforehand, after the service is executed, only if the service is successfully executed, etc. All these different ways are defined by the policies that control the processor's behavior. 


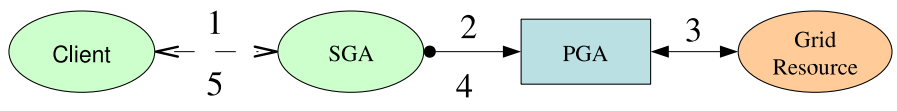

Figure 5. Simple Producer.

\subsubsection{Service Rental}

A slightly more complex control topology is the Service Rental as shown in Figure 6. Here a Social Grid Agent sells or donates the services of one or more of the Production Grid Agents it is in control of. After the social or economic agreement (step 1), the Social Grid Agent instructs the Production Grid Agent to accept requests from the Client with a certain modality (usually token modality, time slot modality, deadline modality or combined modalities) (step 2). The Client is now capable of directly using the Production Grid Agent (steps 3 and 4).

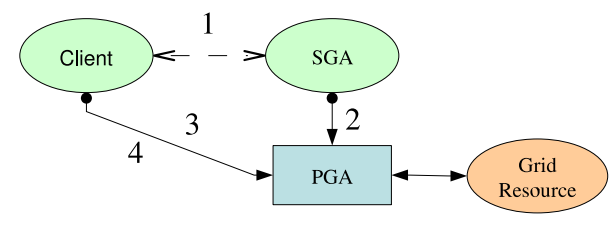

Figure 6. Service Rental.

\subsubsection{A Complex Scenario}

A more complex scenario is represented in Figure 7. Here a client asks for a complex service, the execution of which requires three different grid resources $(\mathrm{a}, \mathrm{b}$ and $\mathrm{c})$. These resources can represent (as in the use case) two different job submission systems and a workflow engine or, in other cases, storage devices and computational power. The Social Grid Agent interacting with the client directly controls only "grid resource a" through one of its Production Grid Agents. When the Social Grid Agent receives the request from the client (step 1) it checks if the other Social Grid Agents with which it has a relation are able to fulfill the services that are needed (step 2 and 3). If this is so, a social arrangement is made to allow the Social Grid Agent to gain control of the Production Grid Agents for the necessary time (step 4). The Social Grid Agent then instructs its Production Grid Agent about the other Production Grid Agents it can use for the completion of the complex service (step 5). The service is then executed and the result is sent back to the client (step 6 and 7).

\subsection{Social topologies}

As for the production topologies where Social Grid Agents control Production Grid Agents, the agents in the
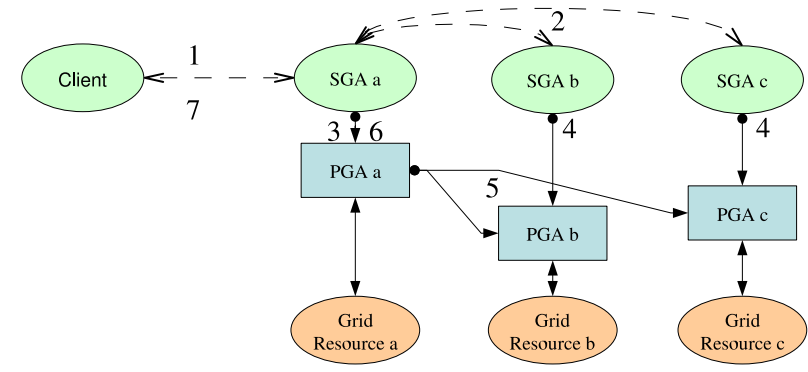

Figure 7. A more complex scenario.

social layer can also be arranged in different structures. In order to achieve this the same design used for the production control is replicated with a few changes. Firstly anyone (either humans or agents) can take control of an agent if he/she/it knows the appropriate startup code. If the code is correct the owner of the Social Grid Agent is set. Owners can set social/economic policies and access rights for controllers. Controllers can set social and economic policies for clients. Such policies encompass the type of purchase to which an actor is entitled, such as: the Free Exchange where services can be used without the need of a payment. This modality is usually present between agents that have cooperative relationships. This modality can also be enforced in Keynesian scenarios, e.g. Standard Purchase where services can be bought for a standard price (this modality is usually the default one and open to all agents) and the Discounted Purchase where services can be bought for a discount price. This modality is usually used in the Keynesian Scenarios and co-operative relationships.

As for the Production Grid Agents, social and economic access modalities can be set by role: the Owners that have complete control over a Social Grid Agent can set access rights for Controllers and Users and they can set social policies; the Controllers that have partial control can set access rights for users and set social and economic policies that control pricing (controllers are usually used to enforce specific policies on a subset of resources for a set of users), and the users that can access the services with the social/economic policies enforced by a controller or owner.

This mechanism allows the implementation of many different social topologies:

\subsubsection{Simple Relationship}

In this Social Topology, such as that in Figure 8, an owner or controller sets the pricing policies of a Social Grid Agent. The Social Grid Agent then responds to the requests of its clients accordingly. 


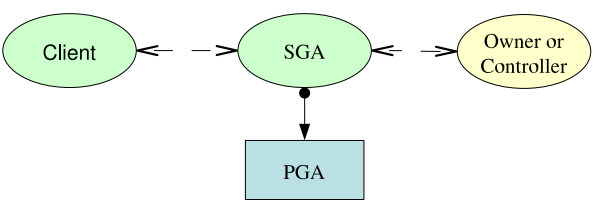

Figure 8. Simple Relationships.

\subsubsection{Tribe}

In a Tribe, such as that in Figure 9, the Social Grid Agents accept the pricing and access policies of the tribe (usually free or discounted prices for all the members of the tribe). Then all Social Grid Agents can use part of the resources of the tribe to fulfill their needs.

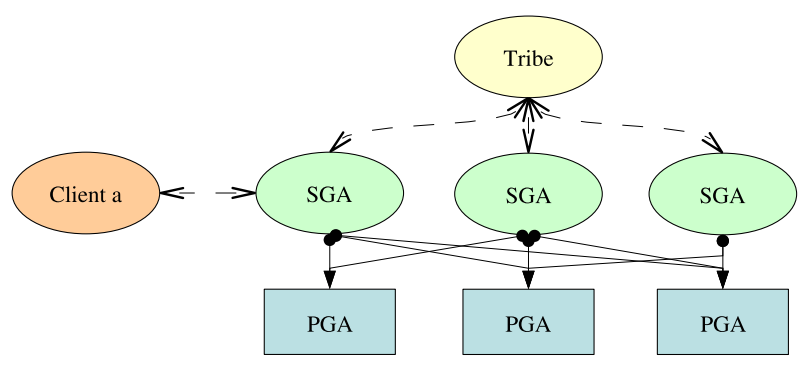

Figure 9. Tribe.

\subsubsection{Keynesian Scenario}

In a Keynesian Scenario, such as that in Figure 10, the Social Grid Agents accept pricing and access policies from an Authority; these pricing and access policies usually refer to a subset of all the clients of the Social Grid Agents.
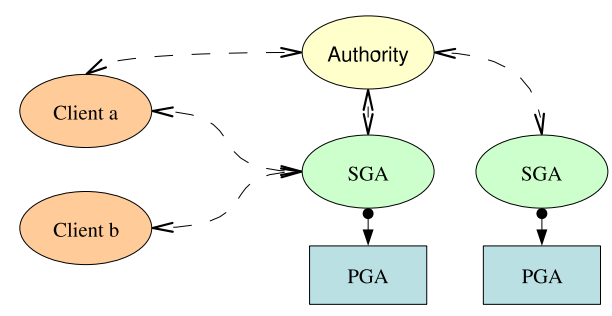

Figure 10. Keynesian Scenario.

\section{The agent's environment: A metagrid}

We now describe the architecture of the environment where the agents "live", see Figure 11. The environment is a metagrid composed from a variety of (otherwise possibly incompatible) components that are made interoperable via the activities of the agents and some basic services. At the center of the architecture is the user, who communicates with all the other components through a user interface.

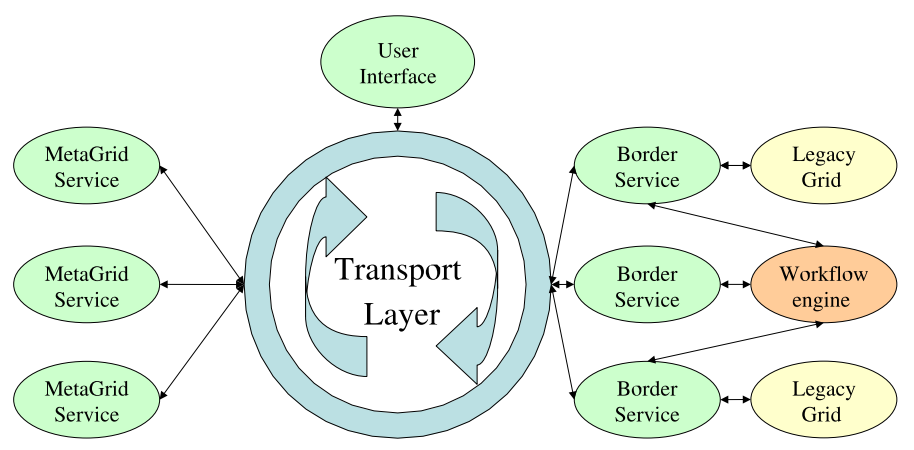

Figure 11. metagrid architecture.

The communication among all the metagrid components is provided by the metagrid Transport Layer that allows the communication between three main groups of metagrid services. The first group is the Middleware Services, on the right of Figure 11, comprising all the existing middlewares that are to be encompassed by the metagrid architecture. These components are divided into two types: workflow middleware such as WebCom[14], and grid middleware such as LCG2 and GT4[1]. Each of these components is extended with an agent (either a Production Grid Agent or a Social plus a Production Grid Agent) that allows communication with the metagrid transport services.

The second group is the Native Metagrid Services, on the left of Figure 11, that comprises those services that were developed to tackle specific metagrid issues. Thus far there are just two of these services: the Metagrid Security eXchange (MSX) service that provides a central market for security credentials, and the Metagrid Job eXchange (MJX), a market where any middleware can exchange job-related actions with any other middleware. Below we briefly describe these components.

\subsection{Transport Layer}

The transport layer is actually two layers, one for control and one for data. The Control Transport Layer is based on Globus GT4. Both Social Grid Agents and Production Grid Agents are able to interact with these layers. LCG2, GT4 and Webcom services have been successfully developed. The Data Transport Layer is for accessing or moving data between other components of the metagrid. It is composed of two different services, the first based on file staging mechanism implemented with gridFTP and RFT, and a second more advanced service based on a transparent grid filesystem [13][7] that allows applications to interact with files as if they were part of the local filesystem. 


\subsection{Border Agents}

Border Agents have the duty to link the agents to existing grid resources. They can link to job submission systems, storage services, information systems or workflow engines. They usually consist of a single Production Grid Agent controlling the existing grid resources under the direct control of some Social Grid Agents in the metagrid or they consist of a couple of Production/Social Grid Agents representing a simple production unit able to sell/donate the services offered by the existing grid resource. Workflow engines, on the other hand, can be modeled in two different ways:

- Monolithic workflow engines where the entire service of the workflow and the services it relies upon is sold or controlled as a single item.

- Open workflow engines that can execute a workflow where every action is just a new message sent to a Production or Social Grid Agent. In this case the added value of this service consits solely in the ability to execute the workflow whilst the services triggered by the workflow may be controlled by other agents.

\subsection{Metagrid Job eXchange}

The Metagrid Job eXchange (MJX) enables any middleware to exchange job-related actions with any other middleware. These actions include job submission and status requests. It is the duty of this service to submit a job, described in the job description language of one middleware, to any other middleware. The Metagrid Job eXchange also offers a Social Grid Agent that has the duty to find the optimal solution for the submitted jobs

\subsection{Metagrid Security eXchange}

The Metagrid Security eXchange (MSX) is a central market that enables different middlewares to trade security credentials in a tightly controlled manner. The KeyNote Credential Authority (KNCA) and Delegation Service are components of the MSX that are particularly important for interoperability between WebCom, which uses KeyNote [4] for authorization, and grid middleware that uses GSI-based security [10].

\section{Use Case}

This use case encompasses three different grids: LCG2, GT4 and WebCom, and two native metagrid services: the Metagrid Job eXchange and the Metagrid Security eXchange. In this example, the Metagrid Job eXchange, the Metagrid Security eXchange and WebCom are directly controlled by the same Social Grid Agent while LCG2 and GT4
Service Providers are controlled by two separate Social Grid Agents with which social and/or economic exchange is to be established to gain the necessary services.

The social topology is described by the upper part of Figure 12. The control/ownership relations are drawn with vertical lines. The production topology is represented in the lower part of Figure 12.

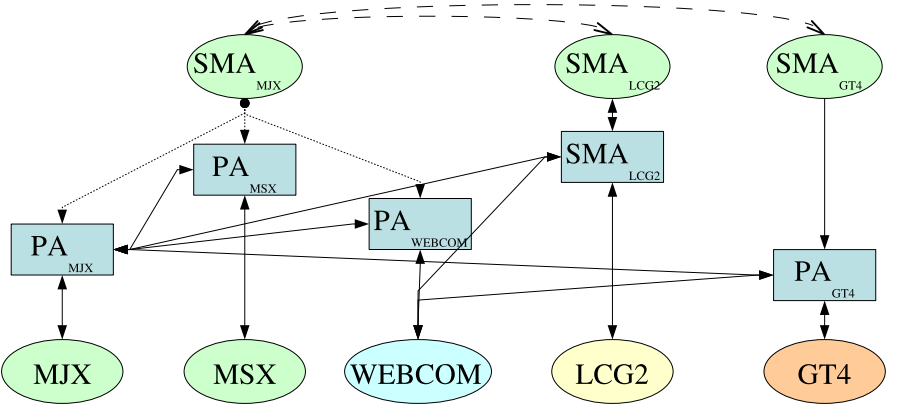

Figure 12. Topology of the use case.

The behavior of the system is as follows:

1. Job submission: The user submits a complex job, the execution of which requires LCG2, GT4 and WebCom resources.

2. Execution planning: The Social Grid Agent $\left(S G A_{M J X}\right)$ that controls the Metagrid Job eXchange Service decides whether to accept to try to submit the job or not

3. Needs evaluation: $S G A_{M J X}$ assesses the needs for the successful submission of the job: these are the Metagrid Security eXchange and LCG2, GT4 and WebCom resources. It then checks this list against the resources it directly controls: the Metagrid Security eXchange and WebCom. This results in a list of the resources that are needed and that must be obtained through social exchange

4. Social and Economic arrangements: $S G A_{M J X}$ checks if amongst the list of other Social Grid Agents it knows there is any that offers the resources it needs. If so it contacts the agents and asks what is the cost of such resources. This cost can be zero for Social Grid Agents with which $S G A_{M J X}$ has cooperative relationships. If the social and economic arrangements can provide enough resources for job execution then the Social Grid Agents that control the LCG2 and GT4 resources $\left(S G A_{L C G 2}\right.$ and $\left.S G A_{G T 4}\right)$ instruct their Production Grid Agents $\left(P G A_{L C G 2}\right.$ and $\left.P G A_{G T 4}\right)$ to accept the requests coming from $S G A_{M J X}$. Currently this is done by setting a token modality access policy for the requests coming from 
$P G A_{M J X}$. The job is then passed to the Production Grid Agent $\left(P G A_{M J X}\right)$ with the list of the other Production Grid Agents that will be needed ( $P G A_{M S X}$, $P G A_{W E B C O M}, P G A_{L C G 2}$ and $\left.P G A_{G T 4}\right)$.

5. Job execution: $P G A_{M S X}$ submits the job to the Production Grid Agent that controls the WebCom resource. WebCom unfolds the workflow graph that represents the job and starts executing it. When a node representing an operation that needs an LCG2 or a GT4 resource is encountered, the job description (JDL or RSL) is sent to the Production Grid Agent that control the resources $\left(P G A_{L C G 2}\right.$ and $\left.P G A_{G T 4}\right)$. This Production Grid Agent allows this execution as it was instructed to do so by its Social Grid Agent (either as Owner or Controller).

6. Job termination: When the complete job has been executed the results are passed back to the Metagrid Job eXchange and thence passed back to the user.

\section{Conclusion}

The first prototype of this combined architecture has proven that the design is sound and flexible enough to cope with different scenarios and different allocation methods. This prototype also shows that the design of the agents allows for the creation of arbitrarily complex social structures that encompass co-operative systems such as Tribes and Keynesian Scenarios and purely competitive systems. Future work will deal with the introduction of a banking system to replace the credit tokens that are now used for the payments, and also the introduction of workflow engines other than WebCom. Finally we will pursue the design of more sophisticated social relations and credit/debit based systems able to allow temporary imbalances in the way resources are consumed (to cope with urgent and unforeseen needs by one or more Social Grid Agents).

\section{References}

[1] Globus toolkit web page.

[2] Social Grid Agents Sourceforge project page.

[3] A. Abraham, R. Buyya, and B. Nath. Nature heuristics for scheduling jobs on computational grids. In The 8th IEEE International Conference on Advanced Computing and Communications (ADCOM 2000), Cochin, India, Dec. 2000.

[4] M. Blaze. Using the KeyNote Trust Management System, March 2001

[5] R. Buyya, D. Abramson, J. Giddy, and H. Stockinger. Economic models for management of resources in peer-to-peer and grid computing. In Technical Track on Commercial Applications for High-Performance Computing, SPIE International Symposium on The Convergence of Information Technologies and Communications (ITCom 2001), Denver, Colorado, USA, Aug. 2001.
[6] R. Buyya, S. Chapin, and D. DiNucci. Architectural models for resource management in the grid. In The First IEEE/ACM International Workshop on Grid Computing (GRID 2000), Bangalore, India, Dec. 2000.

[7] B. Coghlan, G. Quigley, S. Maad, J. Ryan, E. Kenny, and D. OCallaghan. A transparent grid filesystem. In PARA'06 Workshop on State-of-the-Art in Scientific and Parallel Computing, Umea, Sweden, 18 - 21 June 2006.

[8] Enabling Grids for E-sciencE (EGEE), 2006.

[9] E. Elmroth and P. Gardfjll. Design and Evaluation of a Decentralized System for Grid-wide Fairshare Scheduling. In First IEEE Conference on e-Science and Grid Computing, May 2005.

[10] I. Foster, C. Kesselman, G. Tsudik, and S. Tuecke. A Security Architecture for Computational Grids. In ACM Conference on Computers and Security, pages 83-91. ACM-P, 1998.

[11] J. Foster, C. Kasselman, C. Lee, B. Lindell, K. Nahrsted, and A. Roy. A distributed resource management architecture that supports advanced reservations and co-allocation. In Seventh International Workshop on Quality of Service, 1999.

[12] P. Gardfjll, E. Elmroth, L. Johnsson, O. Mulmo, and T. Sandholm. Scalable Grid-wide Capacity Allocation with the SweGrid Accounting System (sgas). 2006. Draft version. Final version to be submitted for journal publication.

[13] S. Maad, B. Coghlan, G. Quigley, J. Ryan, E. Kenny, and D. O'Callaghan. Towards a complete grid filesystem functionality. Future Generation Computer System (Elsevier) Special issue on Data Analysis, Access and Management on Grids, to appear 2006.

[14] J. P. Morrison, J. J. Kennedy, and D. A. Power. WebCom: A Web Based Volunteer Computer. The Journal of Supercomputing, 18:47-61, 2001.

[15] A. Mukherji. Walrasian and Non-Walrasian Equilibria: An Introduction to General Equilibrium Analysis. Oxford University Press, 1990.

[16] G. Pierantoni, E. Kenny, and B. Coghlan. An Architecture Based on a Social-Economic Approach for Flexible Grid Resource Allocation. In Cracow Grid Workshop (CGW05), Cracow, Poland, November 2005.

[17] G. Pierantoni, E. Kenny, and B. Coghlan. An Agent-based Architecture for Grid Societies. In PARA 06, Umea, Sweden, June 2006.

[18] G. Pierantoni, E. Kenny, and B. Coghlan. Social Grid Agents as a Metagrid Technology: An approach for flexible resource allocation in heterogeneous Grid Middlewares. In Cracow Grid Workshop (CGW06), Cracow, Poland, October 2006.

[19] G. Pierantoni, E. Kenny, B. Coghlan, O. Lyttleton, D. O'Callaghan, and G. Quigley. Interoperability using a Metagrid Architecture. In HDPC 06, Paris, France, June 2006.

[20] G. Pierantoni, O. Lyttleton, D. O'Callaghan, G. Quigley, E. Kenny, and B. Coghlan. Multi-Grid and Multi-VO Job Submission based on a Unified Computational Model. In Cracow Grid Workshop (CGW05), Kracow, Poland, November 2005.

[21] J. Sherwani, N. Ali, N. Lotia, Z. Hayat, and R. Buyya. Libra: An economy-driven job scheduling system for clusters. Softw., Pract. Exper., 34(6):573-590, 2004. 